\title{
Real-time Solution via Dynamic Simulation for the Six Degree of
}

\section{Freedom Platform}

\author{
Lizong $\operatorname{Lin}^{1, \text { a, }}$, Xiao $\mathrm{Li}^{2}$,, Xiaopeng $\mathrm{Ni}^{3, \mathrm{c}}$ and $\mathrm{Gang}^{\mathrm{Li}} \mathrm{i}^{4, \mathrm{~d}}$ \\ ${ }^{1,2,3}$ Department of Mechanical Engineering, East China University of Science and Technology, Shanghai 200237, China

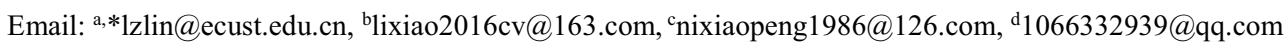

\begin{abstract}
In order to provide a new way for dynamic simulation experiments, a real-time solution for the six degree of freedom platform was developed. The mathematical model of an improved Six-DOF Stewart platform was used to study positive solutions and inverse solutions. According to the parameters of different platforms, different function signals were selected to generate motion control data by using Visual C++ programming. Motion control card was embedded into industrial computer, data was sent automatically to the control card by the program when the platform ran. The output of the control card was analog voltage, and it was amplified to send to the proportional valve, then the flows of six hydraulic cylinders were controlled by the six proportional valves. So a closed-loop control CNC system was formed. The expected action could be realized by the platform. Experiments have proved that the method is simple, efficient and easy to operate. It can not only accompany the implementation moving of relevant actions in the $3 \mathrm{D}$ movie theater, but also provide the signal source for the road spectrum of simulated driving test of the automobile.
\end{abstract}

Keywords. Six DOF platform, positive solutions, inverse solution, visual C++, Googol NC Card.

\section{Introduction}

The 6-DOF motion platform is a very important experiment instrument, which is highly concerned about by most university and research institute in the world, for the simple and compact structure, high moving precision, stiffness, stability, ability of large load endurance [1]. For example, like the flight simulator [2], ship simulator [3], car -driving simulator [4], earthquake simulator, dynamic film and some kinds of device for entertainment. At the same time the platform is also provide a method which is engaged in different types of real-time driving operator. Stewart platform [5] is widely used here: it consists of two boards (up and down), six hydraulic cylinders, six couples of universal hinges (also up and down), the down board is fixed on the base, while the cylinders connect the up board and moving into the postures which we want by the movement of stretching and shrinking.

The precision and real-time property of the 6-DOF platform [6] is greatly dependent on the cylinders' movement, which is determined by the real-time position feedback and calculation. This calculation contains positive solutions and inverse solutions [7]. The positive solution is to get the posture of the platform by collecting the cylinders' amount of displacement, while the inverse solution is to calculate the cylinders' displacement by the posture feedback. In this paper, a mathematical model is made for the Stewart platform, and the solutions are calculated by the computer program. The 6-DOF platform is controlled by the numerical control system based on the PC, and Googol's GTS-800-PV-PCI-G motion controller is used, whose analog output is between $-10 \mathrm{~V}$ to $10 \mathrm{~V}$. The controller can handle almost eight axes. A hardware platform [8] of open CNC system is made by combining the computer and the Googol 
card (controller). In the whole system, the controller is the core, the hydraulic system is the drive unit and the cable encoder is the feedback sensing element. All those parts is made into a semi-closed loop. The implementation of the system is a mode of host computer and the slave computer. The host computer is used to charge the human-computer interaction and the data calculation. And the calculated data is put into the Googol card to realize the expected movement.

\section{Platform structure}

The 6-DOF platform is composed of the upper board, the down board and 6 hydraulic cylinders. The down board is stable and the upper board can move. Both boards are connected by the hydraulic cylinders. The structure of the platform is indicated in Figure 1. Suppose at first the two boards' center line is in the same vertical surface, and the stable coordinate system is named ' $\mathrm{XYZ}$ '. The new position of the board can be calculated by the coordinate transformation matrix [9] in kinematics after the upper board moved.

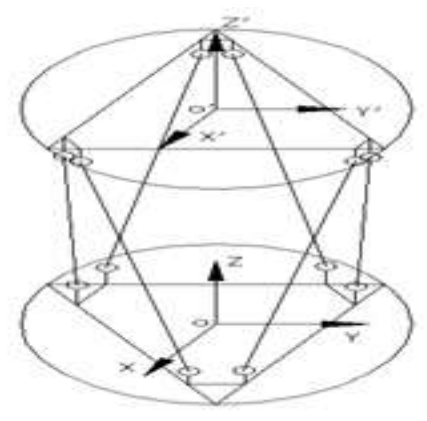

Fig.1. Structure Figure of 6-DOF Platform.

\section{Hardware}

The hardware of the 6-DOF platform mainly contains: the IPC, Googol motion controller, amplifier, encoder, hydraulic cylinders and motion platform. The hardware structure is showed in Figure 2. The pattern of setting communication between the host and the slave computer is used [10], because the IPC is very powerful machines. PC, as the host computer, which is in charge of the interaction between man and the system, and the real-time feedback [11] such as to show the status of the system and the code order input. The Googol NC card, working as the slave computer, can feedback the information of the movement control, which contains the start/stop order, the control of the direction and velocity, and I/O information. The proportional valve and the encoder are set to make up the closed-loop control system. The amplifier is to receive the analog signal released by the NC card, which is amplified and sent to the proportional valve to control the expenditure degree of the valve which constrains the liquid amount. The amplifier gets the feedback signal from the closed-loop control system, and then it sends the signal to the proportional valve to control the hydraulic cylinders. 


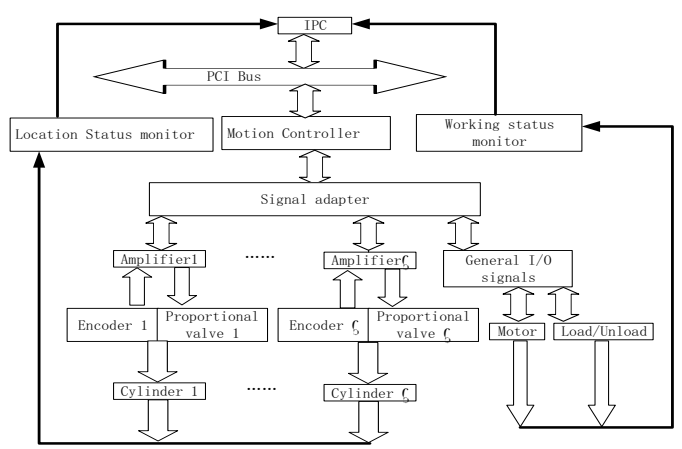

Fig.2. Composition Diagram of System Hardware.

\section{The design of the software to reach the positive solution and the inverse solution}

Considering the tough atmosphere in the factory (vibrations, dust, strong EMI, long running time), Yanhua IPC is selected. The software is based on Windows OS, which has the ability of handling more than one task at the same time, and win32 exe files is used to fit the upper software. The IPC software is made by Visual $\mathrm{C}++6.0$, which is masked by many Windows API, called MFC (Microsoft Foundation Class) library [12]. The users can develop their own programs only by understanding the MFC library and Windows OS. The software is helped by App Wizard and Class Wizard, and set the exe files based on the dialog boxes. In this way, widely used functions is put into the interface to make it convenient to operate.

\subsection{The Realize of the Inverse solution Research Program}

According to the kinematics [13], the Inverse solution is defined as the cylinders' position parameters calculated from the board's position parameters. The relationship between the mathematics model and the closed solution is used to decide the length of the cylinders and get the displacement. This way is simpler than other methods. In the "CsixDofDlg" head files, the cylinder displacement matrix named "DeltaL" (double type) and the parameters of each board's displacement and angle are defined. The principle of Block diagram of anti-solution program [14] is showed in the following Figure 3.

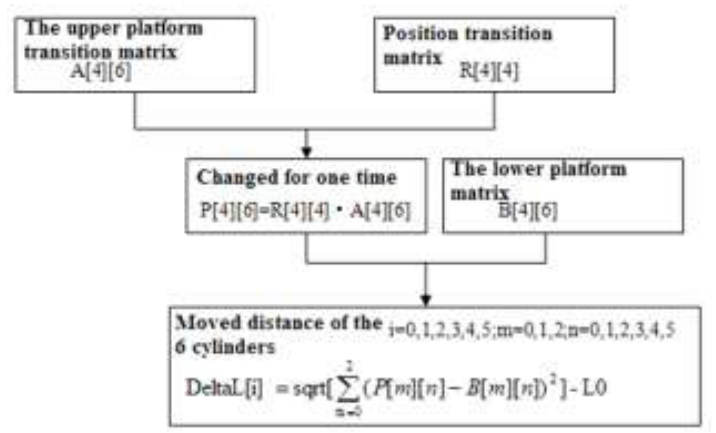

Fig.3. Block Diagram of Anti-solution Program.

The parameters of matrix A and matrix B, and L0 are from Access database, for the board and the cylinder length must be initiated before start. ADO object is used to define a combination and a set pointer: _ConnectionPtr, m_pConnection; _RecordsetPtr; $m \_p$ Recordset. At last the main interface of the inverse solution is showed in Figure 4. 


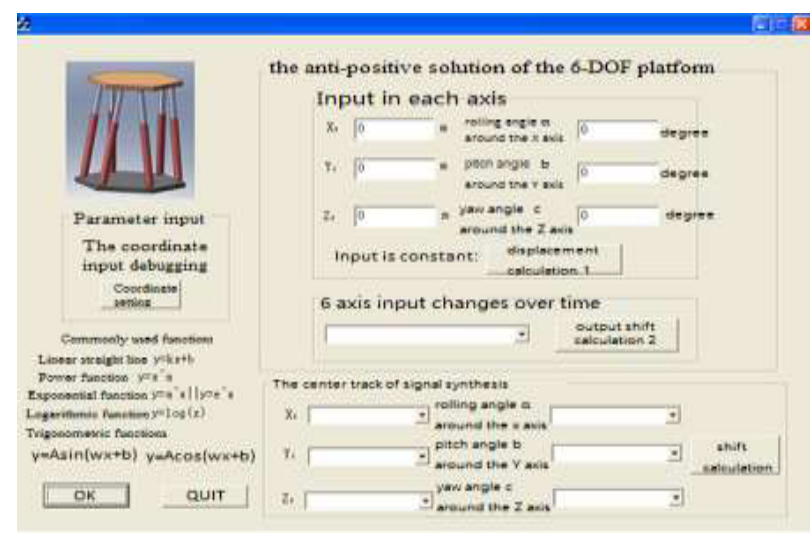

Fig.4. Interface of Platform Signal Generator.

The signal source can be superimposed in any way by using the higher mathematics function and it is easy to create many kinds of data. These data can supply many data for many simulation experiments. Among them the most widely used are the road spectrum and the 3D movie. The collection of road spectrum [15] is to get the wave which the road forces the vehicle to vibrate, and send them to the servo controller after filtered. The conclusion about the parameters of the road such as the flatness can be drawn by collecting the road information from the sensor set in car. With the help of other dynamics simulation software we can also check the flatness of the car, the fatigue of the parts in car, and thus work out the method to relief the car vibration. Collecting the data in real experiment is simple, but it feedbacks messes of data, and the time or road status are limited. However, the 6-DOF platform can realize any movement and movements, Vehicle motion based on the reproducibility of the measured data can also be based on the virtual experimental data vehicles to achieve a variety of road movement. Similarly when 3D movie is played, the platform also can generate the motion and make you feel you are in the movie. All these above are determined by our platform. And the signal source is the key to do these simulations. Appropriate signal source can help to achieve various analog devices motion control. In Figure 5, the signal of the upper board on the $\mathrm{X}$ direction is sin wave, while there is no signal on the other direction. The curves of displacement shown in Figure 5 is calculated by MATLAB.

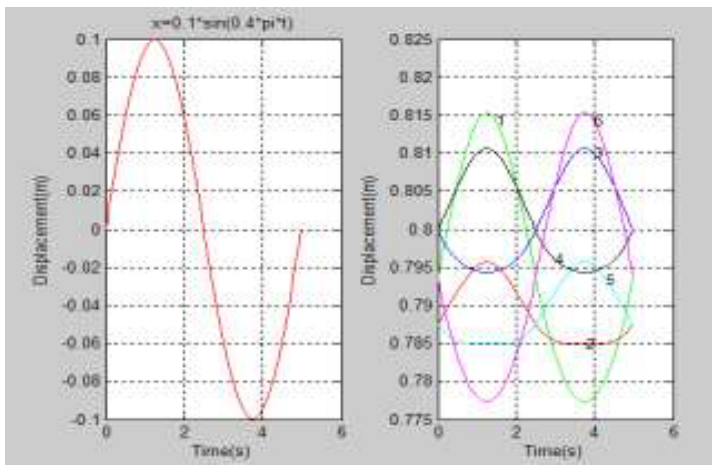

Fig.5. Displacement Curve of Each Cylinder.

\subsection{The Research of the Positive Solution}

According to the kinematics, the positive solution is defined as to get the boards' position parameters when given the cylinders' parameters. It is hard to get the positive solution, so numerical methods such as the Newton method with the anti-solving equations are often used. And the programming time is too long; the large platform is hard to move by the cylinders. In this paper, a small platform model is established shown in Figure 6. The two boards are connected with six 
displacement sensor [16] through hooker hinged. Data acquisition card collects the real-time data from the displacement sensor.

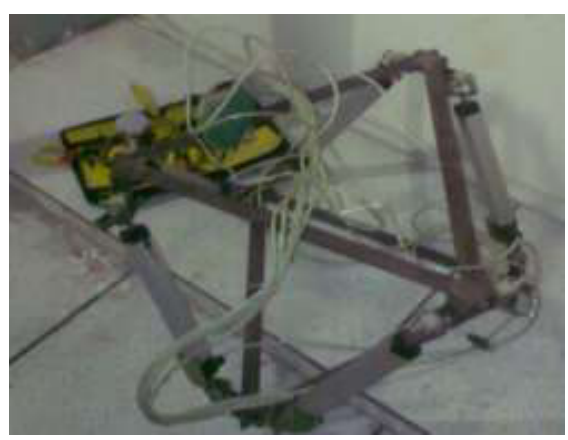

Fig.6. Platform Model.

From the Figure 7 we will know how it is operated. The collected data is stored in PC as txt files, and the first column is time (ms), the data was collected every $200 \mathrm{~ms}$, the second to the sixth column was set for displacement sensors whose maximum capacity is 1023 , and these data is transferred and sent into ACCESS database as shown in Figure 8 .

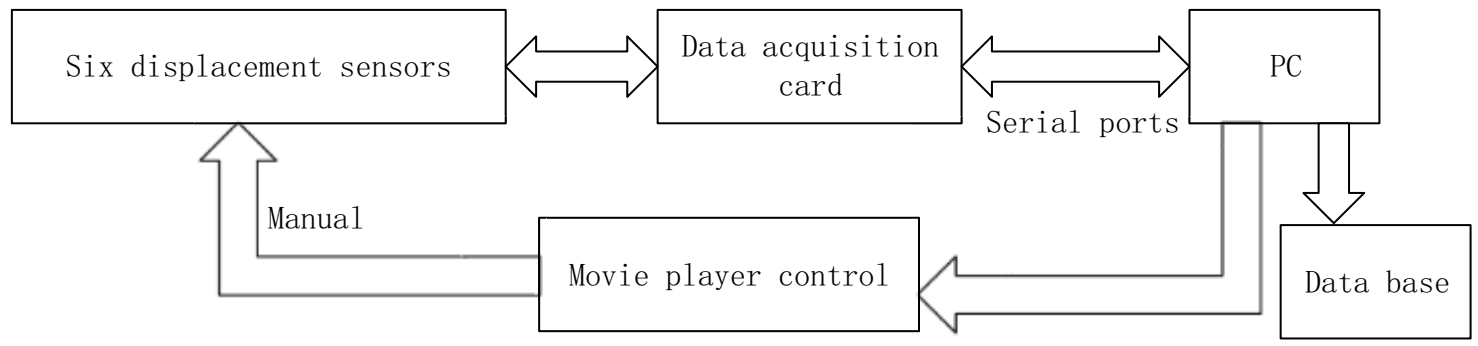

Fig.7. Positive Solutions for Data Acquisition.

\begin{tabular}{|c|c|}
\hline |6 & 6 \\
\hline 266 & 0 \\
\hline 9656 & 6 \\
\hline 606 & 6 \\
\hline 265 & G \\
\hline 1060 & 6 \\
\hline 1265 & 6 \\
\hline 12466 & o \\
\hline 1660 & 6 \\
\hline 1865 & 6 \\
\hline 2606 & c \\
\hline 2200 & $\mathbf{a}$ \\
\hline 22,606 & 6 \\
\hline 2660 & s \\
\hline 2806 & 0 \\
\hline 3606 & 37 \\
\hline 3266 & 55 \\
\hline 3206 & 68 \\
\hline 3606 & 96 \\
\hline 3865 & 12.2 \\
\hline 24665 & 177 \\
\hline 3,296 & $1>7$ \\
\hline 2,24605 & 178 \\
\hline 24656 & 176 \\
\hline 9866 & 186 \\
\hline 5660 & 225 \\
\hline 5260 & 236 \\
\hline 52 & \\
\hline
\end{tabular}
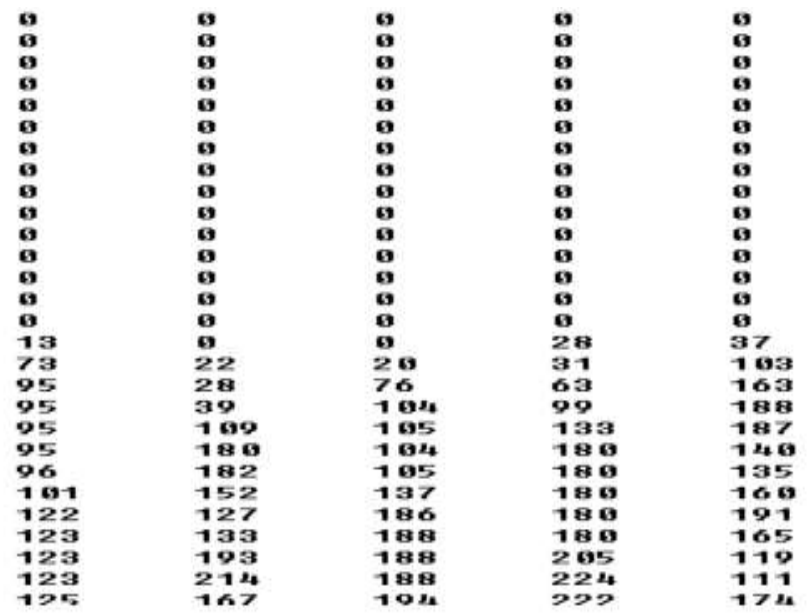

Fig.8. The Collected Data.

\section{Conclusions}


This paper is mainly about the research of the improved Stewart 6-DOF platform in the respect of the positive solution and the inverse solution. A method of getting the signals is proposed and proved on the Googol card system:

(1). IPC + Googol card works, and it can help to improve the research of the 6-DOF platform.

(2). Many kinds of signal sources can be used to mix them into some useful signals. To make the vehicle experiment more convenient, to improve the road spectrum testing, to let the audience of 3D films more comfortable.

(3). $\mathrm{VC}++$ is a very useful and powerful software, and with the Googol card database, there are many functions in it and it does well to NC research.

\section{References}

1. C. Qian, J.Q. E, M. liu, Y.W. Deng, H. Zhu. Six degrees of freedom parallel Stewart platform vibration dynamic model analysis. Hunan Univ. J. (natural science edition). No.2, 36-42 (2016).

2. H. Wang, X.S. Chen. Simulating Six-DOF Motion Platform of Flight Simulator. J. Vol.31, No.5, 778-782 (2012)

3. Y.L. Wang, Y. Lu, J.M. Liang. 6 DOF motion platform location analysis and measurement control in Flight Simulator. Comput. Meas. Control. J. Vol13, No.11, 1243-1244, 1249 (2005).

4. Y.J. Pi, X.Y. Wang, X.H. Luo, X. Gu. Random Wave Spectrum Simulation on Six DOF Ship Motion Simulator. Vib. Testing. Diag. J. 2010, Vol.30, No.4, 75-378 (2010).

5. Q.H. Cao, X.X. Zhang. Car Driving Simulator Telepresence Research and Development on 6 DOF motion platform. Nanchang Inst. Tech. J. Vol.28, No.4, 8-11 (2009).

6. H. Zhao, S.Y. Zhang. Hydraulically Driven of Stewart platform and Trajectory Planning. Mach. Tool. Hydr. J. Vol.35, No.8, 137-139 (2007).

7. D.Y. Yang, L.M. Chen. Six DOF Hydraulic Platform Motion Simulation Based on SolidWorks. Mach. Tool. Hydr. J. Vol.36, No.9, 127-128, 150 (2008).

8. B.F. Cai, C. Liao. Six DOF Stewart-type Platform for Positive and Negative Solution. Science Paper Online. J. http://www.paper.edu.cn: 1-6.

9. L.Z. Lin, G. Lan. G Code System Oriented Googol NC Card. Mold Tech. J. No.1: 5-8 (2009).

10. S.Y. Zhang, H. Zhao, J.W. Han. Real-time Control of 6 DOF Motion Platform Forward / Inverse Solution Algorithm Mach. Tool. Hydr. J. No.3, 133-135 (2010).

11. S.S. Zhu, H.P. Shen, Z. Wang, Y.F. Li, Z.H. Ma. Forward and Inverse Kinematics Solution for 4-3 Type 6-DOF Parallel Mechanism. Changzhou Univ. J. (natural science edition). Vol.25, No.4, 32-36 (2013).

12. J. Wang, Y. Y, S.F. Luo, Z.Y. Li. Development of the Stewart Platform Motion Control System Based on PMAC Controller. Mach. Tool. Hydr. J. Vol.40, No.9, 77-80 (2012).

13. K. Song, R.N. Liu, W.M. Li. Book of Visual C++ Development. People's Posts and Telecommunications Press.. Beijing (2007).

14. L. Li. 6-DOF Parallel Platform Displacement and Control Method. Harbin Engineering University (2008).

15. Z. Q. Yao, X.N. Guo, X.B. Liu. Modeling and Simulation of 6 DOF motion platform inverse position. Fluid Power Trans. Control, No.6, 38-40 (2010).

16. R.P. Wang, X.Z. Jiao, G.L. Wang. Recognition Based on a Typical Road Surface of the Car Body Vertical Acceleration Spectrum. Auto. Eng. J. Vol.30, No.12, 1047-1051 (2008). 